\title{
El turismo de cruceros en Uruguay: determinantes socioeconómicos y comportamentales del gasto en los puertos de desembarco
}

\author{
Recibido: 23 de febrero de2017 - Aceptado: 24 de julio de2017
}

Doi: dx.doi.org/10.12804/revistas.urosario.edu.co/economia/a.6149

\author{
Alicia Bellani* \\ Juan Gabriel Brida* \\ Bibiana Lanzilotta*
}

\section{Resumen}

En Uruguay, como ocurre en otros países latinoamericanos, el turismo de cruceros ha aumentado su importancia como generador de divisas. Con el objetivo de contribuir a la planificación de los programas turísticos e intensificar dicha tendencia, en este trabajo se analizan los distintos determinantes del gasto de cruceristas en los puertos de desembarco de Punta del Este y Montevideo. Las estimaciones de modelos de selección de Heckman sobre las encuestas de los años 2010 a 2014 muestran que son algunas pocas características las que determinan el nivel de gasto: la nacionalidad, viajar en grupo, visitar Montevideo y, particularmente, el grado de satisfacción con el viaje. Este patrón no ha experimentado variaciones significativas en el periodo de análisis.

Palabras clave: gasto de cruceristas, turismo, Uruguay, Heckit.

Códigos JEL: C24, L83

* Grupo de Investigación en Dinámica Económica (GIDE), Facultad de Ciencias Económicas y de Administración, Universidad de la República (Montevideo, Uruguay).

Correspondencia. Correo electrónico: bibiana.lanzilotta@gmail.com

Para citar este artículo: Bellani, A., Brida, J. G., \& Lanzilotta, B. (2017). El turismo de cruceros en Uruguay: determinantes socioeconómicos y comportamentales del gasto en los puertos de desembarco. Revista de Economía del Rosario, 20(1), 71-95.

Doi: dx.doi.org/10.12804/revistas.urosario.edu.co/economia/a.6149 


\title{
Cruise Tourims in Uruguay: Socioeconomic and Behavioural Determinants of Spending at Landing Ports
}

\begin{abstract}
In Uruguay, as in other Latin American countries, cruise tourism has increase its importance as a source of foreign exchange. In order to contribute to the planning of tourism programs and intensify this trend, this paper analyses the socioeconomic, satisfaction and behavioural determinants of cruise passengers at landing ports. Results from Heckman's selection models applied on surveys from 2010 to 2014 show that only a few characteristics determine the level of spending: the Brazilian nationality, travel in groups, visiting Montevideo and being satisfied with the trip. This pattern has not had significant variations in the period.
\end{abstract}

Keywords: Cruise passengers spending, Uruguay, Heckit.

JEL Classification: C24, L83

\author{
O turismo de cruzeiros no Uruguai: determinantes \\ socioeconômicos e comportamentais do gasto nos portos \\ de desembarque
}

Resumo

No Uruguai, como ocorre noutros países latino-americanos, o turismo de cruzeiros tem aumentado a sua importância como gerador de divisas. Com o objetivo de contribuir planificação dos programas turísticos e intensificar dita tendência, neste trabalho analisam-se os distintos determinantes do gasto de cruzeiristas nos portos de desembarque de Punta del Este e Montevideo. As estimações de modelos de seleção de Heckman sobre os inquéritos dos anos 2010 e 2014 mostram que são algumas poucas características as que determinam o nível de gasto: a nacionalidade, viajar em grupo, visitar Montevideo e, particularmente, o grau de satisfação com a viagem. Este patrão não tem experimentado variações significativas no período de análise.

Palavras-chave: gasto de cruzeiristas, turismo, Uruguai, Heckit Classificação JEL: C24, L83

\section{Introducción}

La industria de cruceros ha experimentado un crecimiento sostenido en los últimos 25 años, comenzando la década del noventa con casi 4 millones de pasajeros hasta llegar a más de 23 millones en 2015 (CLIA, 2014). Es el sector más dinámico dentro de la industria del turismo y de las actividades recreativas. Desde 1980 ha experimentado un crecimiento anual de pasajeros de aproximadamente 7,2\% anual (CLIA, 2014) y, según la Organización Mundial de Turismo, entre 2004 y 2014, las vacaciones en cruceros a nivel mundial ha crecido 
aproximadamente $20 \%$ más que otro tipo de vacaciones (Brida et al., 2014a). Gran parte de este crecimiento se debe a los destinos emergentes como Asia y Australia que han experimentado incrementos muy importantes en el número de visitas (24\% y $14 \%$ entre 2014 y 2015, respectivamente) (Brida et al., 2015).

La industria de cruceros beneficia a la economía de destino de una manera similar a la que lo hace otro tipo de turismo receptivo (llegan al destino en avión, reservan noches de hotel y compran bienes y servicios). La diferencia radica en que los turistas que viajan en modalidad de cruceros realizan su gasto principalmente en el crucero, y el origen del crucero es, en la mayoría de los casos, extranjero. Por tanto, el impacto económico de la industria de cruceros se origina en el gasto que realizan los pasajeros en el destino, al desembarcar en el puerto. Dentro de las fuentes más importantes de costos y beneficios se destacan el ingreso de divisas extranjeras que provienen tanto de los turistas como de las empresas de cruceros, la recaudación de impuestos provenientes de cargos al servicio de navegación, los cargos portuarios y otros impuestos que apliquen sobre la empresa de cruceros, el incremento del empleo, etc. El incremento del crucerismo también genera externalidades, por ejemplo, los cruceristas que llegan en avión a la localidad donde tomarán el crucero genera un aumento del tráfico aéreo y este conlleva externalidades negativas tales como la polución y ruido ambiental. Algo similar sucede cuando los cruceristas realizan una visita turística en el destino, lo que puede llevar a congestión del tráfico y del lugar de visita. El propio crucero genera externalidades, en particular, sobre la fauna marina local (Dwyer \& Forsyth, 1998).

Estos costos y beneficios tendrán mayor o menor impacto dependiendo del tipo de puerto del que se trate: puerto de escala o puerto de embarque. En este sentido, un puerto de escala es aquel en donde los pasajeros desembarcan temporalmente y luego embarcan para continuar en el crucero a otro destino. En este caso, el consumo generado por el turismo está relacionado con los tours, shopping y excursiones terrestres. Alternativamente, en un puerto de embarque los pasajeros comienzan y terminan el viaje en crucero. En este tipo de puerto, la estadía en hoteles es el principal gasto de los turistas. (Brida et al., 2014a).

Resulta interesante estudiar las características de la demanda de turistas en la modalidad de cruceros, ya que el impacto que produce el sector en el Turismo y en la economía de un país es muy ventajoso. Nótese que la temporada de cruceros se extiende entre los meses de noviembre a abril de cada año, lo que aporta turistas en los meses de temporada media donde el turismo de sol y playa (principal atractivo para los turistas no-cruceristas) no está en su pico más alto. Estos cruceristas desembarcan en los puertos para realizar otras actividades turísticas además de las de sol y playa, lo que contribuye a 
alargar la temporada turística y atrae visitantes en meses menos identificados con el turismo de sol y playa; por tanto, alienta a los operadores de servicios turísticos a desarrollar actividades nuevas para este tipo de turismo ${ }^{1}$.

En este trabajo se intenta aportar conocimiento sobre el turismo de cruceros en Uruguay, con el objetivo de entender cómo puede el país beneficiarse del dinamismo que está experimentando el sector. Con el objetivo de conocer las características de los turistas que llegan al país en la modalidad de cruceros y cómo ellas inciden en su decisión y nivel de gasto, se intentará identificar los perfiles de turistas de cruceros con base en las características económicas y no económicas (sociales, demográficas, etc.) y cómo estas impactan en el gasto que los turistas realizan. Las características que se utilizaron para construir los perfiles son: edad, género, ocupación, residencia, turista repetitivo, tamaño del grupo, satisfacción en varios ítems como bebida y alimentación, playas, paisaje, tranquilidad, etc., puerto de escala y fecha de visita. Estos factores se trabajarán como variables independientes en el modelo y la variable dependiente será el gasto total. Adicionalmente, se realizará un análisis intertemporal para encontrar evidencia acerca de si existieron o no cambios en el perfil de cruceristas que llegan a Uruguay y si dicha evolución impactó en el gasto que realizan.

El resto del trabajo se organiza del siguiente modo: la sección 2 describe las principales características del turismo de cruceros, tanto a nivel internacional como para el caso particular de Uruguay. Con el fin de conocer los antecedentes sobre el gasto de curceristas en los destinos y las variables que más se han utilizado en esta literatura, en la sección 3 se detallan los principales trabajos revisados sobre el tema y se hace un resumen comparativo de los principales resultados de esta área de investigación. La siguiente sección presenta los datos y la metodología empleados en el estudio. La sección 4 presenta los resultados empíricos del estudio, y la sección final contiene las principales conclusiones del trabajo.

\section{La industria de cruceros}

El turismo de cruceros nace en la década de 1970 y su desarrollo coincide con la transformación de la industria de transporte transatlántico de pasajeros que

1 Nótese que la participación porcentual del mes de marzo en el total anual de turistas arribados aumenta cuando se consideran los turistas totales (es decir, incluidos los cruceristas), pasando de $9,6 \%$ a $10,3 \%$ en 2011 , de $13,6 \%$ a $14,9 \%$ en 2012 , de $9,7 \%$ a $11,0 \%$ en 2013 y de $8,1 \%$ a $10,3 \%$ en 2014 (calculado sobre la base de datos del Ministerio de Turismo y la Dirección Nacional de Migraciones de Uruguay). 
competía con la actividad aérea (WTO, 2010). En lo que se refiere a la demanda, está concentrada en unos pocos países. En 2013, el $90 \%$ de los pasajeros provenían de 10 países: EE.UU. (10,92 millones de pasajeros), Gran Bretaña $(1,73)$, Alemania $(1,69)$, Italia $(0,87)$, Australia $(0,83)$, Canadá $(0,77)$, Brasil $(0,73)$, China $(0,73)$, Francia $(0,52)$ y España $(0,48)$ (CLIA, 2014). Algo similar pasa con la oferta, los destinos se concentran en su mayoría en América del Norte y Europa (CLIA, 2014).

La capacidad de los cruceros medida por medio de la oferta en cantidad de camas también experimentó un dinamismo similar a la demanda de cruceros. La capacidad de los cruceros se incrementó en un 84,2\% desde 2003 a 2013 (CLIA, 2014). Según datos de CLIA en 2014 la tasa de ocupación de los cruceros fue de $100 \%$ y la demanda sigue superando a la oferta. El Caribe y las Bahamas sigue siendo el principal destino de los cruceros, representa más de un tercio de total de destinos que visitan los cruceros. Un estudio realizado por BREA (2015) para la FCCA indicó que para la temporada de cruceros de 2014/2015², el sector generó 3,16 billones de dólares correspondientes a gastos directos, 75050 puestos de trabajo y 976 millones de dólares en sala$\operatorname{rios}^{3}$. El gasto total directo por pasajero incrementó un 7,6\% con respecto al último estudio correspondiente a la temporada 2011/2012 y llegó a 133,78 dólares y, en promedio, el gasto fue de 95,92 dólares por visita. Estos gastos corresponden a las categorías de excursiones, alimentación y compras. El porcentaje de pasajeros que desembarcaron fue de $88 \%$ entre pasajeros y $39 \%$ para los tripulantes. Los principales aspectos de satisfacción fueron los tours, la cortesía de los empleados de las tiendas y la amabilidad de los residentes.

\section{Descripción de la industria de cruceros en Uruguay:}

Uruguay no es ajeno al dinamismo que está experimentando la industria de cruceros mundialmente. Los barcos que llegan al país visitan los puertos de Montevideo y Punta del Este y, en general, sus rutas incluyen el puerto de Buenos Aires (Argentina) y varias ciudades brasileñas. Históricamente, los puertos uruguayos han sido puertos de escala, pero desde el año 2012 el puerto de Montevideo se convirtió en puerto de embarque y desembarque de cruceristas.

2 La temporada comienza en mayo de 2014 y termina en abril de 2015.

3 Los destinos participantes fueron Antigua y Barbuda, Aruba, Bahamas, Barbados, Belice, Bonaire, Islas Vírgenes Británicas, Cabo San Lucas, Islas Caimán, Colombia, Costa Maya, Costa Rica, Cozumel, Curaçao, Dominica, República Dominicana, Ensenada, Granada, Guadalupe, Guatemala, Honduras, Jamaica, Martinica, Mazatlan, Nicaragua, Progreso, Puerto Rico (San Juan), Puerto Vallarta, San Kitts y Nevis, Santa Lucía, San Maarten, Tobago, Trinidad, Turks y Caicos, Islas Vírgenes Americanas. 
Al igual que sucede a nivel mundial, en Uruguay el turismo de cruceros es una pequeña porción dentro de la industria turística. Según datos de CLIA, el crucerismo mundial representa el $2 \%$ del total del mercado de viajes de ocio (CLIA, 2014). Medido en cantidad de pasajeros, también es un sector pequeño; el flujo de turistas mundial fue en total de, aproximadamente, 1,13 billones en 2014, mientras que, como se mencionó anteriormente, la cantidad de pasajeros que hizo turismo en cruceros llegó a 23 millones. En Uruguay, en el año cerrado de 2015 el total de cruceristas alcanzó un 13,11 \% del total de turistas que visitaron Uruguay (Ministerio de Turismo del Uruguay -Mintur-, 2016). En la temporada de 2015-2016 llegaron 161 cruceros al país y desembarcaron más de 400000 personas (Anuario 2016, Mintur). Estos turistas gastaron $11141587 \mathrm{mi}$ llones de dólares, lo que corresponde al 0,6\% de los gastos totales que realizaron los turistas que llegaron al país. El gasto medio per cápita durante 2015 de los pasajeros de cruceros fue de 35 dólares, mientras que el de los turistas fue de 599 dólares (Cálculos propios en base al Anuario 2016, Mintur).

En las tablas 1 y 2 se reportan datos sobre la cantidad de cruceros arribados y el gasto de los cruceristas desembarcados en los puertos uruguayos.

Tabla 1. Cruceros arribados por puerto

\begin{tabular}{lccccc}
\hline \multicolumn{1}{c}{ Puerto } & $2011-2012$ & $2012-2013$ & $2013-2014$ & $2014-2015$ & $2015-2016$ \\
\hline Montevideo & 119 & 122 & 121 & 108 & 105 \\
Punta del Este & 106 & 101 & 116 & 64 & 56 \\
Total & 225 & 223 & 237 & 172 & 161 \\
Variación & & $-1 \%$ & $6 \%$ & $-27 \%$ & $-6 \%$ \\
\hline
\end{tabular}

Fuente: datos publicados en el Anuario de 2014, Minturd.

Se puede verificar que la temporada de 2013-2014 registró un record en varios rubros correspondientes al turismo de cruceros. La cantidad de cruceros arribados llegó a 237, desembarcaron más de 400000 personas y gastaron casi 19 millones de dólares corrientes.

Tabla 2. Gastos de cruceristas desembarcados por puerto

\begin{tabular}{lcccccc}
\hline \multicolumn{1}{c}{ Puerto } & $2010-2011$ & $2011-2012$ & $2012-2013$ & $2013-2014$ & $2014-2015$ & $2015-2016$ \\
\hline Montevideo & 5232921 & 9834529 & 10383173 & 8661802 & 7264605 & 7640237 \\
Pta. del Este & 8058383 & 11049562 & 8516291 & 10484448 & 3678865 & 3501350 \\
Total & 13291304 & 20884091 & 18899464 & 19146250 & 10943470 & 11141587 \\
Variación & & 0,57 & $-0,10$ & 0,01 & $-0,43$ & 0,02 \\
\hline
\end{tabular}

Fuente: datos publicados en el Anuario de 2014, Minturd. 


\section{Antecedentes}

A medida de que el crucerismo va ganando importancia en relación con su rápido desarrollo y su impacto en las economías locales, el interés por estudiar este sector se extiende paralelamente. Este interés se refleja no solamente en el incremento del número de estudios, sino también en los desarrollos metodológicos específicos, con el objetivo de conocer los impactos del sector y mejorar las acciones de los partícipes públicos y privados.

En un estudio realizado para Cartagena de Indias (Colombia), los autores analizan el perfil del crucerista en relación con los gastos que realizan en la ciudad. Encuentran que los grandes consumidores se diferencian del resto porque gastan más en joyas, son más jóvenes, son mujeres residentes en Estados Unidos, viajan por primera o segunda vez en crucero, permanecieron más tiempo en la ciudad y viajan en grupos grandes (Brida et al., 2010). Henthorne (2000), por el contrario, realiza un análisis de factores en Jamaica y concluye que los consumidores de mayor edad gastan más que los más jóvenes. En un trabajo posterior, Henthorne et al. (2013) llegan a la conclusión de que los visitantes que viajan en grupo dedican más tiempo al shopping y compran más. Adicionalmente, encuentran que los visitantes que llegan por primera vez, están más desconformes con el ambiente alrededor de la localidad que visitan que aquellos visitantes que repitieron el viaje $y$, por tanto, el comportamiento de compra se ve deteriorado. Finalmente, hallan que el riesgo percibido y la seguridad tienen un impacto en la intención de volver a Jamaica.

Otro enfoque distinto en el estudio del gasto de los turistas es el de la fidelidad de estos. Tal es el caso de Petrick (2004), que analiza la hipótesis de los beneficios que suponen los turistas más fieles, en el sentido en que estos muestran mayores intenciones de compra, divulgan publicidad boca a boca y son más sensibles al precio en comparación con los turistas que visitan por primera vez o aquellos menos fieles. El resultado del estudio ratifica esta hipótesis y encuentra que los turistas que visitan por primera vez y los menos fieles tienen menor sensibilidad al precio y gastan más. De manera similar, el trabajo de Parola et al. (2014) corrobora la asociación positiva entre la satisfacción que experimenta el crucerista en el destino visitado y el gasto que realiza en este, y además, la relación también positiva entre el nivel de satisfacción y el deseo de volver al mismo destino.

Para el caso de Uruguay, se realizaron dos estudios con el objetivo de conocer el gasto de los cruceristas que llegan a los puertos de Montevideo y Punta del Este. En uno de ellos, Brida, J. G., Bukstein, D. y Tealde, E. (2015) estiman dos regresiones de sección cruzada y demuestran que el tamaño del 
grupo y la movilidad dentro del país son las variables más importantes para explicar el gasto. En el trabajo realizado por Brida, J. G., Scuderi, R. y Seijas, M. N. (2014), mediante un modelo Heckit (Heckman, 1979), encuentran que la nacionalidad del pasajero es la principal característica que define el gasto del individuo. Además, hallan que el proceso de decisión de gastar de los cruceristas en la ciudad que visitan es más intuitivo que el que sugiere la literatura para el gasto turístico general. Siguiendo esta línea, Risso (2012) también encuentra que la nacionalidad explica en gran medida el gasto que realizan los cruceristas en los puertos uruguayos. En particular, encuentra que el turista argentino reduce la probabilidad del gasto por persona mientras que el brasileño la aumenta. La probabilidad de gasto es alta (más de $80 \%$ ) y el desagrado por los precios reduce esta probabilidad.

También para Uruguay, Abbruzzo et al. (2014) estudian los determinantes del gasto del turismo receptivo mediante un análisis de modelos gráficos. A diferencia de la mayoría de los estudios, encuentran un rol marginal de las variables sociodemográficas como determinantes del gasto y una importancia directa del gasto con el tipo de alojamiento, el destino y la duración de la estadía. Algunos trabajos comparan el gasto de cruceristas con respecto al del turista tradicional o turista de estadía. Por ejemplo, Larsen et al. (2013) llegan a la conclusión de que el turista de cruceros realiza la mayor parte del gasto dentro del crucero y por tanto realiza un gasto mucho menor que el turista tradicional en la ciudad que visita. Igualmente, aclaran que si se estudia el gasto por hora, el nivel resultante de ambos tipos de turista se equipara. Bresson y Logossah (2011), con el objetivo de estudiar la complementariedad del turismo de estadía y el de cruceros, llegan a un resultado similar para el caso de varias islas del Caribe. Encuentran que ambos tipos de turismo son sustitutos y que es el turismo de estadía, y no el crucerismo el que permite a estos países lograr sus objetivos de crecimiento económico. Más aún, encuentran que desde la década del noventa surgió una "trampa de desplazamiento" del turismo de cruceros sobre el turismo de estadía. El riesgo que identifican los autores es la debilidad negociadora que enfrentan los hacedores de políticas locales con respecto a los operadores de cruceros en una industria oligopolística, lo que da como resultado un incremento del flujo de cruceristas en detrimento del turismo tradicional. Van Beukering et al. (2015) consideran que, si bien, la mayor parte de los turistas que llega a Bermudas lo hace en cruceros, el gasto que estos realizan es ínfimo comparado con el que hace el turismo tradicional (el crucerista gasta el $9 \%$ de lo que lo hace el turista de estadía). Además, el excedente del productor proveniente del turismo tradicional es el doble que el del crucerismo. Visto que 
no se cuenta con datos suficientes, en este trabajo no se adoptará esta línea de investigación, se deja para un trabajo futuro.

Se desprende de la revisión de la literatura que el tema de estudio es relevante y de reciente desarrollo y que cada vez gana más interés en el plano académico. Este trabajo intenta aportar conocimiento a esta literatura especialmente en lo que respecta al estudio de la evolución de las características del crucerista que desembarca en el puerto, con el objetivo de entender su dinámica de gasto y sus preferencias. Es interesante notar que el estudio sobre el gasto de turistas que llegan en crucero aún no tiene un paradigma claro, en el sentido de que no hay un lineamiento establecido en cómo se debe estudiar el tema ni se tiene un estudio global del crucerismo, sino que cada investigador aporta y analiza parcialmente el tema.

\section{Datos y metodología}

En este trabajo se utilizaron cinco bases de datos que surgen de encuestas anuales que realiza el Ministerio de Turismo de Uruguay a los cruceristas que desembarcan en los puertos de Montevideo y Punta del Este. Estas encuestas corresponden a los años 2010-2011, 2011-2012, 2012-2013, 2013-2014 y 20142015, entre los meses de noviembre a abril. Los cruceristas encuestados se seleccionan por el método del muestreo probabilístico; primero realizaron una selección aleatoria de la lista de cruceros que se esperaba iban a llegar (systemic sampling) y luego los entrevistados pertenecientes a grupos de turistas se seleccionaron de manera tal, que se pudiera asegurar la equiprobabilidad (Ministerio de Turismo y Deporte, 2015).

El cuestionario aplicado en la recolección de los datos consta de una serie de preguntas acerca de las características sociodemográficas del crucerista tales como edad, género, país de residencia y ocupación. También se considera la cantidad de veces que ya visitó el país. Otro grupo de características refiere al viaje que realizan e incluye variables como el tamaño y la composición del grupo en el que viajan, el puerto en el que desembarcan, el gasto individual (total y luego desglosado en transporte, alimentación y bebidas, compras y otros). Por último, se pregunta sobre el agrado que sintió el turista acerca de algunos aspectos de la visita tales como, el paisaje edificios, higiene, cordialidad, tranquilidad, playas y otros.

Las bases de datos utilizadas tienen la ventaja de incluir una muestra grande de cruceristas y reportar información sobre una gran cantidad de individuos y para diversas temporadas. Sin embargo, la propia encuesta tiene 
limitaciones en cuanto a información que sería útil pero que no se incluye. Por ejemplo, datos de ingresos de los encuestados, de educación, de gustos, de tiempo fuera del crucero, de comportamientos en el puerto y otras variables sobre la característica del viaje.

La tabla 3 describe las variables a ser utilizadas en este estudio, recabadas a partir de la información de los cuestionarios. En este trabajo se decidió agrupar algunas características de los cruceristas para simplificar el manejo de las variables. Este fue el caso de la variable 'residencia', que fue agregada en regiones, salvo para los casos de Brasil y Argentina. Algo similar se realizó para las variables de Agrado, Edad y Ocupación. Con respecto al Tamaño del grupo se decidió trabajar con las variables ‘viaja solo’ y ‘tamaño del grupo' que corresponde a la cantidad de personas que viajan junto con el encuestado.

Tabla 3. Variables utilizadas en el estudio

\begin{tabular}{lll}
\hline & \multicolumn{1}{c}{ Variables } & \\
\hline Residencia & Edad & Ocupación \\
Brasil & Menor 35 & Ama de casa \\
Argentina & Menor 30 & Empresario \\
Latinoamérica & Entre 36-64 & Profesional \\
América del Norte & Entre 31-59 & Empleado \\
Europa & Mayor 65 & Estudiante \\
Otros & Mayor a 60 & Jubilado-Pensionista \\
Agrado & Género & Tripulante \\
Gusta Act. Turísticas & Hombre & \\
Gusta Ambiente & Mujer & Gasto Total \\
Gusta Zonas Tur. & Tamaño del Grupo & Gasto Transporte \\
Mes & Viaja Solo & Gasto Comidas y bebidas \\
Noviembre & Total Personas & Gasto Compras \\
Diciembre & & Otros Gastos \\
Enero & & \\
Febrero & & \\
Marzo & & \\
Abril & & \\
\hline
\end{tabular}

Fuente: elaboración propia sobre la base de Encuestas Cruceros, Minturd. 
En lo que se refiere a la metodología de análisis, la forma más común en que se ha estudiado el tema es mediante el enfoque clásico de la regresión lineal utilizando estimaciones de mínimos cuadrados ordinarios. Este acercamiento ha tenido grandes críticas, ya que asume que el gasto turístico está distribuido de forma normal a lo largo de toda la recta real, lo cual es incorrecto. Esta metodología puede llevar a estimaciones inconsistentes.

Las metodologías de estimación alternativas que contemplan el hecho de que el gasto es una variable censurada en cero y que su distribución presenta a menudo una asimetría positiva son básicamente dos. La primera alternativa busca obtener estimaciones consistentes, al suponer que el gasto depende de una variable que asume valores positivos cuando el gasto es mayor a cero y cero en el resto de los casos. Estas estimaciones se pueden llevar a cabo mediante una regresión Tobit (Tobin, 1958) que asume una dependencia lineal entre la variable dependiente y sus regresores más un término aditivo de error. En el trabajo de Brida et al. (2015) se mencionan 14 trabajos que siguen esta alternativa. Otra posibilidad es utilizar modelos en dos etapas, donde primero se modela la decisión de gastar o no y luego cuánto se gasta.

En este trabajo se decidió analizar el proceso de decisión del gasto turístico utilizando un modelo en dos etapas. En particular, siguiendo la estrategia del trabajo Brida et al. (2015), se decidió aplicar un modelo del tipo de Heckman, ya que este sortea las restricciones que supone utilizar un modelo Tobit. Este último asume que la probabilidad de que un individuo decida gastar y el nivel de su gasto son determinados por el mismo conjunto de variables e ignora la naturaleza del proceso de decisión en dos etapas. Es decir, asume que la variable explicativa que incrementa la verosimilitud de consumir en la primera etapa de decisión (si gastar o no) también incrementa el nivel de gasto en la segunda. Adicionalmente, utilizando este modelo se capturó el hecho de que el gasto es una variable censurada en cero, es decir, toma valores positivos incluyendo el cero pero nunca va a revelar valores negativos (ver Anexo Método Heckit).

El modelo Heckit de este trabajo se construyó de la siguiente manera: en la primera etapa del modelo (etapa del Probit), donde se determinan las condiciones bajo las cuales el individuo decide si gasta o no, se incluyeron las siguientes variables de satisfacción: gusta actividades turísticas (edificios, playas, cuero, paisaje, casino, casapueblo, tiendas y rutas), gusta zonas turísticas (Montevideo, Mercado del Puerto, Punta del Este, Ciudad Vieja, Dedos, otras zonas turísticas), gusta ambiente (tranquilidad, higiene y gente), con el precepto de que un crucerista satisfecho aumenta su probabilidad de gastar (ver Homburg et al., 2005; Fornell et al., 2010; Moore et al., 2015 y las relativas referencias en estos trabajos). También se incluyó la ocupación 
del crucerista excluyendo a los tripulantes, ya que estos se comportan — con respecto al gasto en el destino - de una forma diferente al crucerista (Brida et al., 2015). Dentro de la categoría 'residencia', se incluyeron en esta etapa del análisis las de los países con mayor número de cruceristas entrevistados, que en este caso fueron Brasil y Argentina con más del 70\% del total de turistas. En ambas etapas se incluyeron la edad, el género, el puerto de desembarque, si viajaba solo y el total de personas en el grupo. Por último, en la segunda etapa se utilizaron, además de las variables mencionadas anteriormente, las de 'tripulante', 'mes de visita' y 'todas las residencias'.

\section{Resultados empíricos}

\subsection{Estadística descriptiva}

La tabla 4 ofrece una síntesis estadística de las variables de interés. Como es posible observar, no ha habido grandes cambios en lo que se refiere a las características de los cruceristas que llegan al país.

Tabla 4. Estadística Descriptiva. Datos, variables y resultados para las distintas temporadas

\begin{tabular}{lccccc}
\hline \multicolumn{1}{c}{ Variable / Temporada } & $2010-2011$ & $2011-2012$ & $2012-2013$ & $2013-2014$ & $2014-2015$ \\
\hline Género & & & & & \\
Femenino & 59,55 & $63, .56$ & 61,83 & 61,08 & 56,71 \\
Masculino & 40,45 & 36,44 & 38,17 & 38,92 & 43,29 \\
Edad & & & & & \\
Hasta 35 & 5,26 & 5,66 & 5,93 & 4,78 & -- \\
Hasta 30 & -- & -- & -- & -- & 4,34 \\
$36-64$ & 76,28 & 77,82 & 74,08 & 81,50 & -- \\
$31-59$ & -- & -- & -- & -- & 74,77 \\
$>65$ & 18,44 & 16,52 & 20,00 & 13,72 & -- \\
$>60$ & -- & -- & -- & -- & 20,89 \\
Ocupación & & & & & \\
Jubilado/Pensionista & 28,70 & 22,64 & 26,08 & 22,75 & 23,45 \\
Ama de casa & 3,91 & 3,99 & 3,50 & 3,17 & 2,44 \\
Estudiante & 1,76 & 2,12 & 1,91 & 2,28 & 1,61 \\
& & & & & Continúa
\end{tabular}




\begin{tabular}{|c|c|c|c|c|c|}
\hline Variable / Temporada & 2010-2011 & 2011-2012 & $2012-2013$ & 2013-2014 & 2014-2015 \\
\hline Empresario & 12,19 & 14,32 & 11,66 & 14,50 & 18,97 \\
\hline Profesional & 35,18 & 34,77 & 33,94 & 35,87 & 31,99 \\
\hline Empleado & 7,78 & 11,03 & 11,66 & 12,01 & 13,68 \\
\hline Tripulante & 7,13 & 6,87 & 6,20 & 5,89 & 3,04 \\
\hline Otros & 3,35 & 4,26 & 5,05 & 3,53 & 4,82 \\
\hline \multicolumn{6}{|l|}{ Mes } \\
\hline Noviembre & 2,86 & 0 & 2,98 & 4,98 & 1,49 \\
\hline Diciembre & 18,31 & 20,03 & 15,35 & 19,08 & 19,68 \\
\hline Enero & 32,79 & 32,55 & 34,69 & 26,84 & 34,79 \\
\hline Febrero & 27,37 & 24,76 & 26,54 & 22,60 & 28,17 \\
\hline Marzo & $18 ; 04$ & 20,22 & 19,17 & 22,46 & 15,86 \\
\hline Abril & 0,63 & 2,44 & 1,28 & 4,04 & 0 \\
\hline \multicolumn{6}{|l|}{ Residencia } \\
\hline Brasil & 31,72 & 40,86 & 45,31 & 43,95 & 56,96 \\
\hline Argentina & 31,86 & 34,58 & 26,36 & 26,32 & 21,44 \\
\hline Am Latina & 4,65 & 5,42 & 3,82 & 4,27 & 3,58 \\
\hline USA/Canadá & 14,52 & 8,14 & 12,10 & 10,11 & 8,30 \\
\hline Europa & 13,30 & 8,92 & 9,49 & 13,13 & 8,19 \\
\hline Otras & 3,96 & 2,08 & 2,92 & 2,22 & 1,53 \\
\hline Primera Vez de Visita & 64,48 & 65,90 & 70,25 & 70,61 & 67,49 \\
\hline \multicolumn{6}{|l|}{ Puerto Desembarque } \\
\hline Montevideo & 40,94 & 47,24 & 51,03 & 46,94 & 60,45 \\
\hline Punta del Este & 59,06 & 52,76 & 48,97 & 53,06 & 39,55 \\
\hline \multicolumn{6}{|l|}{ Gustos } \\
\hline Bebida y Comida & 6,14 & 5,15 & 3,90 & 3,90 & 4,12 \\
\hline Zonas Turísticas & 14,87 & 11,12 & 13,34 & 11,84 & 17,38 \\
\hline Ambiente & 39,55 & 42,07 & 37,07 & 34,67 & 33,79 \\
\hline Actividades Turísticas & 44,35 & 44,93 & 39,45 & 40,58 & 33,44 \\
\hline Tamaño del Grupo (media) & 2,37 & 2,41 & 2,25 & 2,26 & 2,82 \\
\hline Gasto Total (media) & 112,52 & 142,33 & 103,29 & 105,98 & 32,97 \\
\hline Gasto Tour (media) & 7,25 & 10,03 & 8,34 & 9,67 & 2,02 \\
\hline
\end{tabular}




\begin{tabular}{lccccc}
\hline Variable / Temporada & $2010-2011$ & $2011-2012$ & $2012-2013$ & $2013-2014$ & $2014-2015$ \\
\hline Gasto Alimentos (media) & 24,10 & 31,52 & 25,25 & 27,04 & 8,97 \\
Gasto Transporte (media) & 2,17 & 2,84 & 2,93 & 2,83 & 1,09 \\
Gasto Shopping (media) & 72,01 & 93,06 & 58,56 & 58,87 & 18,77 \\
Otros Gastos (media) & 6,99 & 4,87 & 8,21 & 7,57 & 2,12 \\
\hline
\end{tabular}

La mayoría de los cruceristas son mujeres (60\%) que están en su edad media (entre 30 y 70 años). Profesionales, jubilados y pensionistas y empresarios son las ocupaciones de la mayor parte de los cruceristas y normalmente viajan en grupos de entre 2 y 3 personas. La llegada de los cruceros coincide con la temporada alta de verano, que corresponde a los meses de enero y febrero en el hemisferio sur. También es considerable la llegada de cruceros en diciembre y marzo, es decir al comienzo y fin del verano. En la última temporada se puede verificar una caída significativa en la llegada de cruceros en los meses de marzo y abril. La mayor parte de los cruceristas son residentes de Brasil y Argentina, los primeros han aumentado en cantidad a lo largo de las temporadas y los últimos han disminuido su número. Otros residentes de América Latina se han mantenido estables en la llegada a nuestros puertos y visitantes europeos y norteamericanos han disminuido en cantidad. Cerca del $70 \%$ de los cruceristas llega por primera vez a los puertos uruguayos y descienden tanto en Montevideo como en Punta del Este, aproximadamente, en la misma proporción. Se identifica una fuerte caída de visitantes a Punta del Este en la última temporada de cruceros. Los cruceristas están satisfechos con la gente, la tranquilidad y la higiene y también gustan de las actividades turísticas. En lo que se refiere a la evolución del promedio del gasto, se puede observar un pico de gasto en la temporada 2011-2012 y luego una disminución del gasto a lo largo de las temporadas. Finalmente, la tabla muestra que los rubros más importantes de gasto son en alimento y shopping.

\subsection{Resultados empíricos}

Las tablas 5 y 6 reportan los resultados del modelo Heckit para cada una de las temporadas. La tabla 5 presenta resultados para las temporadas 2010-2011 a 2013-2014 y la tabla 6, para la temporada 2014-2015. La razón por la cual los resultados se informan en tablas diferentes es por el cambio en la estructura de la encuesta de cruceristas, en lo que se refiere a los rangos de edad. 


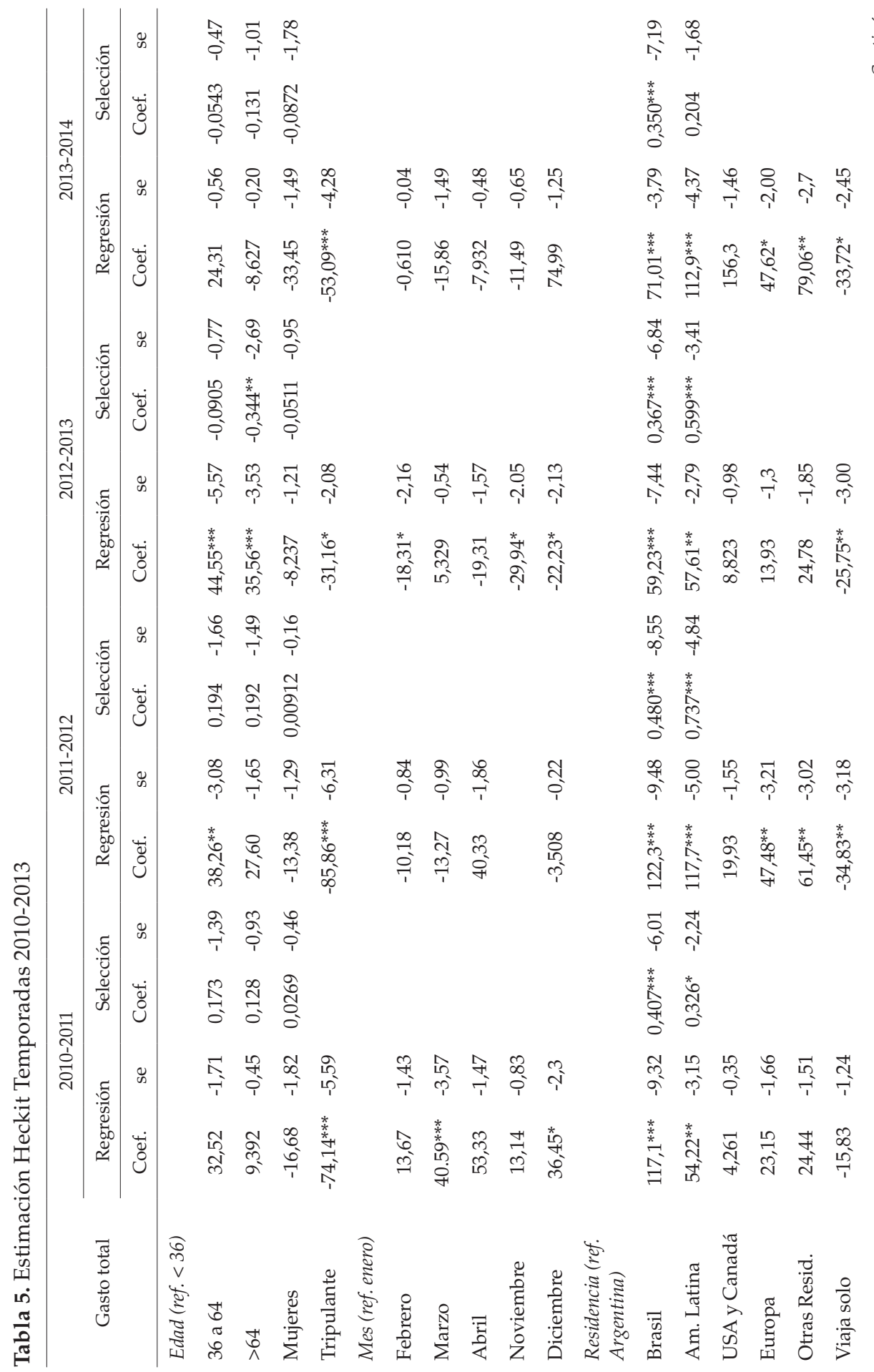




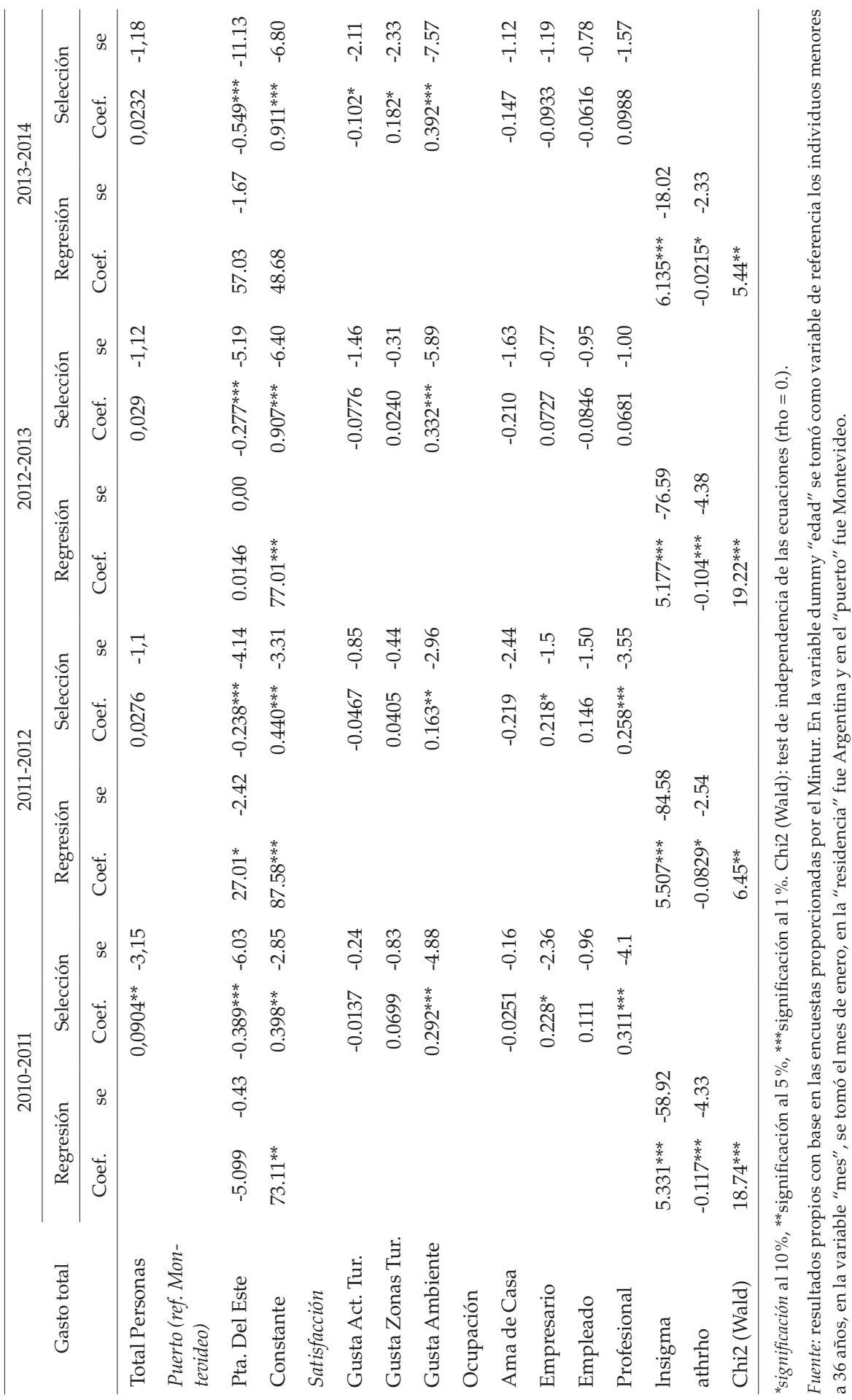


Tabla 6. Estimación Heckman Temporada 2014

\begin{tabular}{|c|c|c|c|c|}
\hline \multirow{3}{*}{ Gasto total } & \multicolumn{4}{|c|}{ 2014-2015 } \\
\hline & \multicolumn{2}{|c|}{ Regresión } & \multicolumn{2}{|c|}{ Selección } \\
\hline & Coef. & SE & Coef. & $\mathrm{SE}$ \\
\hline \multicolumn{5}{|l|}{ Edad $(r e f .<29)$} \\
\hline 32 a 44 & 9,124 & $-1,88$ & 0,0556 & $-0,41$ \\
\hline 45 a 59 & $18,30^{* * *}$ & $-3,32$ & 0,0413 & $-0,29$ \\
\hline$>60$ & $13,34^{* *}$ & $-2,62$ & & \\
\hline Mujeres & $-0,421$ & $-0,11$ & $-0,271^{* * *}$ & $-4,84$ \\
\hline Tripulante & $-15,00^{*}$ & $-2,30$ & & \\
\hline \multicolumn{5}{|l|}{ Mes (ref. enero) } \\
\hline Febrero & 0,896 & $-0,21$ & & \\
\hline Marzo & 4,655 & $-1,13$ & & \\
\hline \multicolumn{5}{|l|}{ Abril } \\
\hline Noviembre & 8,143 & $-0,58$ & & \\
\hline Diciembre & 8,859 & $-1,47$ & & \\
\hline \multicolumn{5}{|c|}{ Residencia (ref. Argentina) } \\
\hline Brasil & $15,74^{* * *}$ & $-3,91$ & $0,418^{* * *}$ & $-7,24$ \\
\hline Am. Latina & $62,96^{*}$ & $-2,38$ & $0,866^{* * *}$ & $-4,92$ \\
\hline USA y Canadá & $18,34^{* * *}$ & $-3,87$ & & \\
\hline Europa & $20,01^{* * *}$ & $-3,57$ & & \\
\hline Otras Resid. & $23,44^{* *}$ & $-2,59$ & & \\
\hline Viaja solo & $23,47^{* * *}$ & $-4,04$ & & \\
\hline Total Personas & & & 0,00599 & $-0,21$ \\
\hline \multicolumn{5}{|c|}{ Puerto (ref. Montevideo) } \\
\hline Pta. del Este & $-4,506$ & $-1,29$ & $-0,292^{* * *}$ & $-5,15$ \\
\hline Constante & $15,83^{*}$ & $-2,4$ & $0,342^{*}$ & $-2,1$ \\
\hline \multicolumn{5}{|l|}{ Satisfacción } \\
\hline Gusta Act. Tur. & & & $0.120^{*}$ & -1.99 \\
\hline Gusta Zonas Tur. & & & $0.199 * *$ & -2.58 \\
\hline Gusta Ambiente & & & $0.371^{* * *}$ & -6.21 \\
\hline \multicolumn{5}{|l|}{ Ocupación } \\
\hline Ama de Casa & & & -0.139 & -0.84 \\
\hline
\end{tabular}




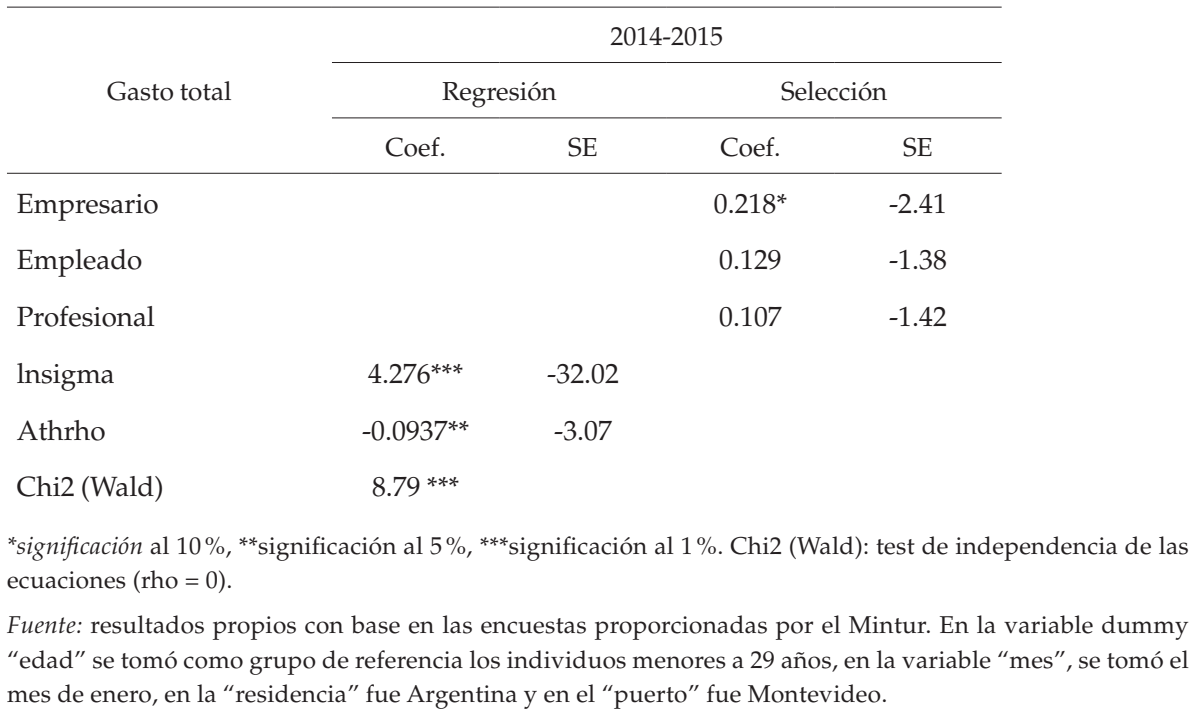

Los resultados de las estimaciones sugieren que las variables clave a la hora de explicar el gasto de los cruceristas fueron la residencia, el puerto y la satisfacción con el ambiente.

La residencia argentina se asocia con un menor gasto y demás baja frecuencia en comparación con personas de otras residencias. Es interesante notar la evolución que ha tenido el gasto de los brasileños. En las primeras temporadas analizadas, el coeficiente estimado para la residencia brasileña es el más alto, destacándose la temporada de 2011-2012 la que registró el mayor impacto (ser brasileño implicó un gasto de 122 dólares más que un argentino). Sin embargo, a lo largo del tiempo esta diferencia se debilita y se constata una distribución algo más equitativa del gasto entre los cruceristas de diferentes residencias (en la temporada 2014-2015 el gasto fue significativo para todas las residencias y mayor al gasto que hicieron los argentinos). El gasto de un brasileño pasó a ser solamente 15 dólares mayor al de un argentino en la temporada 2014-2015. Estos resultados podrían estar respondiendo a la coyuntura política y económica en la que se ha visto inmerso este país en los últimos años. Más allá de la reducción en el nivel de gasto, la probabilidad de gastar de un brasileño sigue siendo la más alta (10 punto porcentuales en promedio para las temporadas analizadas) llegando a 12,3 puntos porcentuales en la temporada 2014-2015.

Históricamente, se evidencia que la frecuencia de gasto de los cruceristas que desembarcan en el puerto de Punta del Este es inferior a la de aquellos que desembarcan en el de Montevideo. Los resultados parecen indicar que el impacto promedio en todas las temporadas analizadas implica que des- 
embarcar en Punta del Este disminuye la probabilidad del gasto en 9 puntos porcentuales. No se encontraron resultados significativos con respecto al nivel del gasto en un puerto y otro. Resultados similares se encontraron en el trabajo de Brida et al., 2014b.

La satisfacción que el crucerista experimenta como resultado de su visita aumenta la probabilidad de que estos gasten en el puerto. Para el caso de Uruguay, la satisfacción con el ambiente que incluye la tranquilidad, higiene y la gente influyó positivamente en la frecuencia del gasto en todas las temporadas (en 2014-2015 implicó una probabilidad de gasto de 11,6 puntos porcentuales). Adicionalmente, en los últimos años se evidencia que la satisfacción con las actividades turísticas y las zonas turísticas también explicaron la probabilidad de gasto de un crucerista. Se encontraron resultados similares en los trabajos de Parola et al. (2004), Brida et al. (2014a) y Risso (2012).

Se observa que la edad, en la mayoría de las temporadas, no parece explicar el comportamiento del gasto de los cruceristas; solamente se encontraron valores significativos para las temporadas 2011-2012, 2012-2013 y 2014-2015 en donde los cruceristas de mediana edad gastaron más que los jóvenes. Este resultado se asemeja con los trabajos de Abruzzo (2014) y de Brida y otros (2014b) que encuentran un rol marginal de las variables sociodemográficas como determinantes del gasto. Si bien, el poder de explicación de la edad no se puede generalizar a lo largo de las temporadas analizadas, se podría decir que los cruceristas más jóvenes son los que gastan menos, resultado que se asemeja al trabajo de Henthorne (2000) y el de Risso (2012). El género tampoco resultó ser una variable que influya en el comportamiento del gasto, aunque en la última temporada se evidencia que las mujeres tuvieron una frecuencia de gasto significativamente menor que los hombres. Este resultado concuerda con otro realizado para Uruguay (Brida et al., 2014b), pero no concuerda con en realizado para Colombia (Brida, Bukstein, Garrido, Tealde \& Zapata, 2010). Viajar solo resultó ser una característica significativa a la hora de explicar la dimensión de lo que gasta un crucerista y su impacto fue negativo. Por el contrario, el total de las personas del grupo no parece influir sobre la frecuencia del gasto en las últimas temporadas. Viajar en grupo implica un mayor nivel de gasto per cápita pero no se pueden sacar conclusiones acerca de una relación incremental entre la cantidad de personas del grupo y su impacto en el gasto. De estos resultados, se podría concluir que viajar en grupo implica un mayor nivel de gasto per capita, resultado que es intuitivo y concordante con otros trabajos analizados (Brida et al., 2010; Henthorne et al., 2013; Brida et al., 2015). Los cruceristas que son empresarios y profesionales son aquellos que gastan con mayor frecuencia. Este resultado se evidencia en todas las temporadas excepto en 2012-2013 y 2013-2014. Los resultados parecen 
indicar que las ocupaciones que a primera vista retribuyen a los cruceristas con mayores ingresos les permiten gastar con mayor frecuencia. Dado que la ocupación del crucerista impacta directamente sobre su ingreso disponible, este resultado parece ser bastante intuitivo, aunque sería deseable conocer directamente el nivel de ingreso de los cruceristas para poder corroborar y evaluar cómo impacta la variable ingreso en el gasto que estos realizan.

En lo que se refiere al mes en el que llega el crucero, se encontraron efectos variados. En la temporada de 2010-2011, se gastó más en los meses de baja temporada en comparación con el mes de enero. En 2012-2013, en noviembre, diciembre y febrero se gastó menos que en enero. En las últimas temporadas se evidencia que el mes dejó de explicar el comportamiento del gasto de los cruceristas. Varios trabajos revisados encontraron que en los meses de baja temporada los cruceristas gastaron más, sin embargo, para el caso de Uruguay no hay resultados concluyentes (Morales \& Carrasco 2014; Brida et al., 2014b). Por último, se indica que el test de Wald que analiza la independencia de las regresiones fue significativo en todas las temporadas, lo que confirma la pertinencia de utilizar el modelo de Heckman en dos etapas.

\section{Conclusiones}

Este trabajo considera el gasto de los cruceristas para realizar un análisis económico de los costos y beneficios de los cruceros que llegan a Uruguay, identificando las variables que afectan ese gasto. Los resultados permiten la estimación del gasto promedio para un pasajero de cruceros típico que arriba a Uruguay y hace posible proyectar los cambios en el gasto en turismo cuando cualquiera de las características significantes cambia, así como ayuda a comprender la probabilidad de gasto en diferentes categorías dependiendo de un conjunto de variables socio demográficas.

En lo que se refiere al gasto, se pudo verificar un pico de gasto en la temporada 2011-2012 y luego una evolución negativa en el resto de las temporadas. Ello podría responder al descenso de turistas llegados en la modalidad de cruceros al país, pero también podrían estar relacionados con el encarecimiento del país en términos reales con casi todas las regiones, en particular con los países vecinos. Otro fenómeno a destacar es el cambio de preferencia en los puertos de desembarque. El puerto de Punta del Este era el preferido por los cruceristas; sin embargo, en la última temporada fueron más las personas que desembarcaron en Montevideo. Esto puede deberse a la particularidad del puerto de Punta del Este, que no es un puerto de cruceros sino que los cruceristas llegan a la ciudad en un barco de menor porte 
desde el crucero, lo que dificulta el desembarque. Esta característica puede estar impactando negativamente en la motivación de los turistas para visitar ese puerto y se estima que el turismo de cruceros en Punta del Este crecería si un puerto para estos barcos fuera construido. Parece plausible realizar un análisis costo-beneficio que permita entender la viabilidad de esta iniciativa, pero esto está más allá del alcance del presente trabajo.

En términos generales, el trabajo muestra que las mayorías de las características sociodemográficas no parecen influir en las decisiones de gasto de los cruceristas, y que las principales variables que explican el gasto fueron la residencia, el hecho de no viajar solo, el puerto de desembarque y la satisfacción. El perfil de crucerista que impacta positivamente en el gasto se puede caracterizar del modo siguiente: es un brasileño empresario o profesional mayor de 30 años que no viaja solo, desembarca en el puerto de Montevideo y está satisfecho con la experiencia. Por el contrario, la categoría de crucerista que impacta en un menor gasto es un crucerista argentino, menor de 30 años que desembarca en Punta del Este. Estos perfiles resultan interesantes para definir políticas económicas orientadas a mejorar la experiencia de viaje y aumentar el gasto de los cruceristas que forman el grupo del perfil positivo. Adicionalmente, se podrían explorar los aspectos que impactan negativamente en el gasto de los cruceristas del perfil negativo para crear programas específicos que motiven a este grupo a realizar un mayor gasto. Los resultados también parecen sugerir que campañas de comunicación y marketing en los países vecinos podrían beneficiar en mayor medida al país como resultado de un mayor nivel de gasto de los cruceristas.

En cuanto a la evolución del gasto, los resultados muestran que en las primeras tres temporadas, el gasto de un brasileño fue mayor que el del resto de las nacionalidades. Sin embargo, este aspecto fue perdiendo influencia en la explicación del gasto, dando lugar a que otras residencias tuvieran mayor impacto en e. Parecería que turistas de otras residencias de América Latina y otras residencias como las asiáticas han estado influyendo en la decisión del gasto en mayor medida en las últimas temporadas. Este resultado estaría indicando que un cambio en el perfil del crucerista, en lo que se refiere a su residencia, se asocia con un cambio en el monto del gasto. Esta evolución sugeriría que se deberían revisar los programas de marketing y de política económica actuales, en el sentido en que si se verifica una evolución en el perfil de crucerista que llega a Uruguay, las decisiones tomadas por hacedores de política y empresarios turísticos deberían acompañar esta evolución.

En términos generales, el trabajo muestra que el crucerismo en Uruguay es un sector muy dinámico del turismo y, por su creciente importancia, debiera ser el foco de más estudios para mejorar las decisiones gerenciales y de políticas 
públicas asociadas con este sector. Con base en los resultados de este trabajo, se pueden sugerir algunas políticas que permitan mejorar los beneficios del turismo de cruceros en Uruguay. Dado que el tamaño del grupo con el que el visitante viaja es una variable poderosa y explica el comportamiento del gasto individual, los empresarios y los encargados de políticas públicas deberían promocionar el Uruguay como un destino con instalaciones apropiadas para las actividades en grupo, como entretenimiento y eventos culturales. En la misma línea, el sector de turismo de cruceros debería promocionar Uruguay como destino en vez de un puerto de Uruguay en particular. Dado que hay un grupo importante de visitantes que repiten la visita a las ciudades, los operadores turísticos deberían pensar en el desarrollo de nuevos productos que pueden inducir a los pasajeros a estar más tiempo en el destino.

Algunas extensiones a este trabajo podrían incluir el análisis de los cruceristas que no gastaron, esto para crear paquetes de actividades y programas que sean adecuados a su perfil. También se podría analizar al crucerista que no bajó del crucero y comparar el gasto del turista de estancia y del crucerista que llega a Uruguay. Otra línea de análisis de interés sería comparar el gasto que realiza un crucerista en Uruguay con el que realiza en otros destinos del mismo crucero. Y, por último, desde el punto de vista metodológico, se podrían comparar el modelo de selección de Heckman con otros, como los modelos Tobit u otro modelo semiparamétrico y contrastar los resultados obtenidos.

\section{Referencias}

Abbruzzo, A., Brida, J. G., \& Scuderi, R., (2014). Determinants of individual tourist expenditure as a network: Empirical findings from Uruguay. Tourism Management 43, 36-45.

BREA (Business Research and Economic Advisors) (2015). Economic contribution of cruise tourism to the destination economies. A survey-based analysis of the impracts of passenger, crew and cruise line spending. (Tesis de Universidad, ciudad). Recuperado de file:/ / C:/Users/alici_000/SkyDrive/Tesis\%20Crucerismo\%202015/Paper/2015-cruiseanalysis-volume-1.pdf

Bresson, G., \& Logossah, K. (2011). Crowding-out effects of cruise tourism on stay-over tourism in the caribbean: Non-parametric panel data evidence. Tourism Economics 17(1), 127-158

Brida, J. G., Bukstein, D., Garrido, N., Tealde, E., \& Zapata-Aguirre, S. (2010). Cruise passengers expenditure in the Caribbean port of call Cartagena 
de Indias: A cross-section data analysis. Estudios y Perspectivas de Turismo 19, 607-634.

Brida, J. G., Monterubbianesi, P. D., \& Zapata Aguirre, S. (2013). Análisis de los factores que influencian el gasto de los turistas culturales: el caso de los visitantes de museos de Medellín. Revista de Economía del Rosario 16(1), 147-168.

Brida, J. G., Scuderi, R., \& Seijas, M. N. (2014a). Segmenting cruise passengers visiting Uruguay: a factor-cluster analysis. International Journal of Tourism Research 16, 209-222.

Brida, J. G., Fasone, V., Scuderi, R., \& Zapata, A. S. (2014b). ClustOfVar and the segmentation of cruise passengers from mixed data: some managerial implications. Knowledge-Based Systems, 70, 128-136.

Brida, J. G., Bukstein, D., \& Tealde, E. (2015). Exploring cruise ship passenger spending patterns in two Uruguayan ports of call. Current Issues in Tourism, 18(7), 684-700.

CLIA (2014). Cruise Lines International Association - The Global Economic Contribution of Cruise Tourism 2013. Recuperado de http: / / www.cruising.org/ docs/default-source/research/global_cruise_impact_analysis_2013. pdf?sfvrsn=2

Fornell, C., Rust, R. T., \& Dekimpe, M. G. (2010). The effect of customer satisfaction on consumer spending growth. Journal of Marketing Research, $47(1), 28-35$.

Heckman, J. (1979). Sample selection bias as a specification error. Econometrica, 47(1), 153-161.

Henthorne, T. L. (2000). An Analysis of Expenditures by Cruise Ship Passengers in Jamaica. Journal of Travel Research, 38(3), 246-250.

Henthorne, T. L., George, B. P., \& Smith, W. C. (2013). Risk Perception and Buying Behavior: An Examination of Some Relationships in the Context of Cruise Tourism in Jamaica. International Journal of Hospitality and Tourism Administration, 14(1), 66-86.

Homburg, C., Koschate, N., \& Hoyer, W. D. (2005). Do satisfied customers really pay more? A study of the relationship between customer satisfaction and willingness to pay. Journal of Marketing, 69(2), 84-96.

Larsen, S., Wolff, K., Marnburg, E., \& Øgaard, T. (2013). Belly full, purse closed: Cruise line passengers' expenditures. Tourism Management Perspectives, $6,142-148$.

Ministerio de Turismo (2016). Anuario 2016. Recuperado de http:/ / www. mintur.uy/index.php/pt/feed-ministerio/item/237582-anuario-2016. 
Ministerio de Turismo y Deporte (2015). Anuario 2014. Recuperado de http:/ / www.mintur.uy/index.php/pt/feed-ministerio/item/235256-anuario-2014

Moore, S. A., Rodger, K., \& Taplin, R. (2015). Moving beyond visitor satisfaction to loyalty in nature-based tourism: a review and research agenda. Current Issues in Tourism, 18(7), 667-683.

Petrick, J. F. (2004). Are loyal visitors desired visitors? Tourism Management, $25,463-447$.

Parola, F., Satta, G., Penco, L., \& Persico, L., (2014). Destination satisfaction and cruiser behaviour: The moderating effect of excursion package. Research in Transportation Business \& Management, 13, 53-64.

Risso, W. A. (2012). El gasto de los cruceristas en Uruguay 2008 - 2010. PASOS Revista de Turismo y Patrimonio, 10(3), 393-406

Van Beukering, P., Sarkis, S., Van Der Putten, L., \& Papyrakis, E. (2015). Bermuda's balancing act: The economic dependence of cruise and air tourism on healthy coral reefs. Ecosystem Services, 11, 76-78.

WTO (2010). World Tourism Organization - Cruise Tourism Current Situation and Trends. Recuperado de http://www.e-unwto.org/doi/ book/10.18111/9789284413645

\section{Anexo Método de Heckman}

En la primera etapa del Heckman se estima un modelo de probabilidad de gasto usando un modelo Probit:

$$
\begin{gathered}
\mathrm{y}^{*}{ }_{1}=\mathrm{X}_{1} \beta_{1}+\varepsilon_{1}, \varepsilon_{1} \sim \mathrm{N}\left(0, \sigma^{2}\right)(\mathrm{v}) \\
\mathrm{y}^{*}{ }_{1}=1, \text { si } \mathrm{y}_{1}>0 \\
\mathrm{y}^{*}{ }_{1}=0, \text { si } \mathrm{y}_{1} \leq 0(\mathrm{vi})
\end{gathered}
$$

donde $\mathrm{y}_{1}^{*}$ es una variable latente, $\mathrm{y}_{1}$ es la variable observada que vale uno si el individuo efectuó un gasto y cero en otro caso, $X_{1}$ es el vector de variables independientes y $\beta_{1}$ es el vector de coeficientes asociado con las variables independientes; $\varepsilon_{1}$ es el término de error.

En la segunda etapa se estima un modelo del tipo:

$$
\mathrm{y}_{2}^{*}=\mathrm{X}_{2} \beta_{2}+\varepsilon_{2}(\mathrm{vii})
$$




$$
\begin{gathered}
\mathrm{y}^{* 2}=\mathrm{y}_{1}, \text { si } \mathrm{y}_{1}>0 \\
\mathrm{y}_{2}^{*}=0, \text { si } \mathrm{y}_{1}{ }_{1} \leq 0 \text { (viii) } \\
\mathrm{y} 2_{2} \mathrm{X}_{2} \beta_{2}+\sigma_{12} \lambda\left(\mathrm{X}_{1} \overline{\beta_{1}}\right)+\mu_{2}(\mathrm{ix})
\end{gathered}
$$

donde $\mathrm{y}_{2}^{*}$ es una variable latente que equivale a $\mathrm{y}_{1}$ (variable que reporta el gasto individual) cuando $\mathrm{y}_{1}{ }_{1}$ es mayor a cero y cero en otro caso; el conjunto de variables explicativas $\mathrm{X}_{2}$ no tiene por qué coincidir con el de la etapa uno, indicando que la decisión de gastar no está determinada por las mismas variables que la decisión del nivel de gasto; $\overline{\beta_{1}}$ son las estimaciones de $\beta_{1}$ derivadas del modelo Probit de la etapa uno y $\mu_{2}$ esel término de error.

Al utilizar una muestra truncada para la etapa dos, se llega a un problema de sesgo de selección, es decir a una correlación entre $\varepsilon_{1}$ y $\varepsilon_{2}$, que produce estimaciones de Mínimos Cuadrados Ordinarios inconsistentes. Por esta razón es que Heckman aumenta la ecuación de la etapa dos por medio de una variable omitida; dicha variable omitida es el inverso del ratio de Mill's (IMR, por sus siglas en inglés): $\lambda\left(X_{1} \overline{\beta_{1}}\right)$ :

$$
\lambda=\varphi\left(\mathrm{X}_{1} \beta_{1}\right) / \Phi\left(\mathrm{X}_{1} \beta_{1}\right)(\mathrm{x})
$$

$\lambda$ se obtiene de la estimación del modelo Probit, calculando el ratio entre el valor de la función de densidad normal estándar $(\varphi)$ con respecto a la función de distribución acumulada normal estándar $(\Phi)$. El inverso del ratio de Mill's se utiliza como instrumento para corregir el sesgo de selección y aproxima una variable que representa la influencia inobservable en la decisión de gastar. Si $\lambda$ resulta significativo implica que hay sesgo de selección y que se ha corregido. 
